\title{
New Standardized Framework for Developing Mobile Applications (Based On Real Case Studies and CMMI)
}

\author{
Ammar khader almasri
}

\begin{abstract}
- the software processes play a vital role for delivering a high quality software system that meets the user's needs. There are many software development models which are used by most system developers, which can be categorized into two categories (traditional and new methodologies). Mobile applications like other desktop applications need appropriate and well-working software development process. Nevertheless, mobile applications have different features which limit their performance and efficiency like application size, mobile hardware features. Moreover, this research aims to help developers in using a standardized model for developing mobile applications.
\end{abstract}

Keywords - software process model, mobile application development, agile approaches

\section{Introduction}

A software process model is an summary of the structural design of the software process [1].The software process is an important factor for delivering a high quality software system that meets the user's needs. Process model is an ordered sequence of activities undertaken to manage, develop and maintain software systems to achieve the project objectives.

A software development process, also known as a software development life cycle (SDLC), SDLC is a process of building software systems[2]. There are many software development models which are used by most system developers, which can be categorized into two categories as shown in figure 1.

Ammar Khader Mohammad Almasri

Amman College/Albalqa Applied University

Jordan

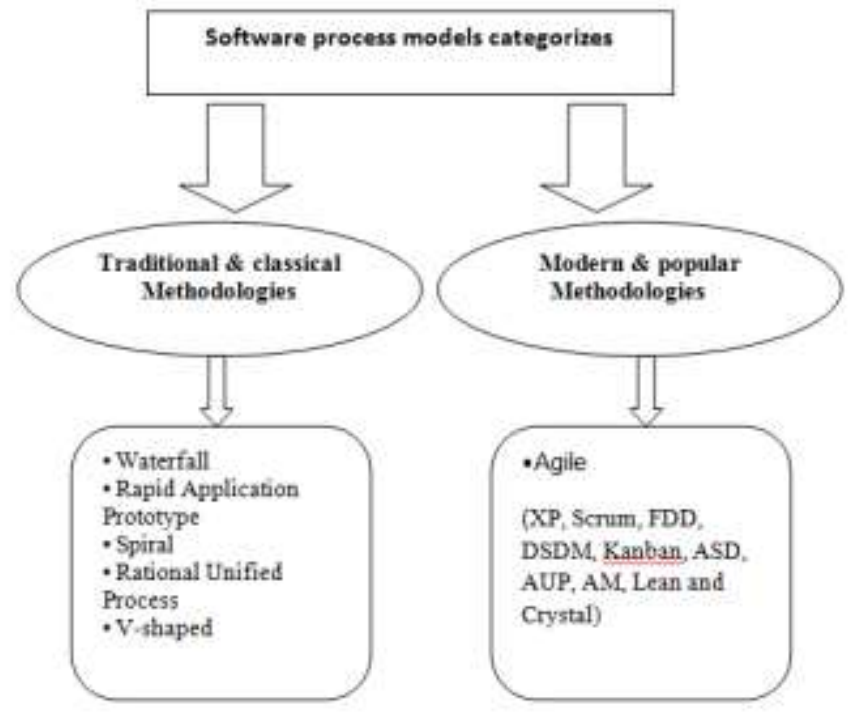

Figure 1. categories of software process models

\section{A. Traditional \& classical Methodologies}

Software methodologies like Waterfall, Rapid Application Prototype, Spiral, Rational Unified Process, and V-shaped are called traditional software development methodologies and these are called the heavyweight methodologies [3]. These methodologies are based on a sequential series of steps.

\section{B. Agile Methodology}

Agile development methods referred to lightweight approaches [4]. Agile development is used the incremental and iterative work strategy, the development life cycle is divided into smaller parts as well as revisited over and over again. It iteratively improves software based on customer feedback [2]. 
There are a number of software development methodologies that have been categorized under the Manifesto umbrella. XP, SCRUM, DSDM, FDD, and Crystal Methods are among the most popular examples of these agile methods.

\section{Agile or traditional methods?}

Before developing any project it is important for a development team to identify some characteristics like critical of project, requirement nature, and size of project to select a software development model that best suits the project. According to [5], the author summarized A comparative analysis for traditional and agile methodologies the result indicate that:

- Traditional methods are used for:

1) highly critical projects

2) requirements do not change often,

3) limited requirements with limited features,

4) it is designed for large projects (Large number of developers)

- Where Modern methodologies are used for:

1) low critical projects

2) requirements changes often and Acquired iteratively

3) Flexibility of design,

4) improvement in quality

5) Iterative and Incremental delivery

6) Increased Performance,

7) Easy fault detection capabilities.

8) It is suitable for medium and small project

9) Customer involvement will lead to a highly Customer Satisfaction

According to [6], Agile models are used to build a project if the project has the following aspects: (1) project's requirement changes frequently (2) project is small (3) needs to deliver product in short period time .

\section{Problem statement}

The key success factor to deliver any software products with high quality outcome is picking up appropriate and well-working software development process[7]. Moreover, mobile applications like other desktop applications need appropriate and well-working software development process.

Nevertheless, mobile applications have different features which limit their performance and efficiency like application size, mobile hardware features (memory size, $\mathrm{CPU}$ clock rate, platform type, data representation, and battery life).

1) Developers for mobile application software face the following challenges:

2) a dynamic environment with frequent modifications in customer needs and expectations[8].

3) technical constraints associated with mobile systems [8].

4) different technological platforms, including different Operating Systems (OS) and `software development platforms [9].

Many studies have been suggested a solution to the above challenges, it has been suggested that Agile is a fit model for the development of mobile applications [10].Moreover, There are a set of characteristics were developed by [11] regarding using agile methodology for mobile application development as follow :(1) High environment volatility(2)Small development teams (3) Identifiable customer (3) Object oriented development (4) Non-safety critical Software (5)Application level Software(6) Small systems (7) and Short development cycles.

\section{Related studies}

Many studies have been deployed to prove that the agile methods can be implemented in mobile applications development .as a result; we found many agile approaches, as discussed below.

A study was conducted by Abrahamsson, et al. [12] in 2004 was the first attempt to integrate agile model with the development process for mobile applications . He developed an agile development approach called Mobile-D. The Mobile-D approach is a development methodology inspired 
on Extreme Programming, Crystal Methodologies and Rational Unified Process (RUP) from four case studies. This approach consists of five phases Explore, Initialize, Productionize, Stabilize and System Fix in sequentially arranged. The result indicated that Mobile-D approach is recommended to be used by a small, co-located team, working in a short development cycle.

Mobile Application Software development based on Agile Methodology (MASAM) was developed by Jeong, et al. [13]. This approach was developed based on Extreme Programming, Agile Unified Process, RUP and the Software and Systems Process Engineering Meta-model. The structure of MASAM confirms a strong tie with Mobile-D. This model consisted by 4 phases Preparation, Embodiment, Development and Commercialization. This methodology does not include any case study.

According to literature review, there is no research studies that provides detail reasons to use Agile as essential model for mobile application development [8] for that reason, this research aims to encourage and contribute to prove that as well as helping developers in using a standardized model for developing mobile applications .

\section{Research Methodology}

1) This research aims to propose a high quality software process model for mobile application development based on agile development methods. So, the research study will go through the six steps as follow:

2) Based on Literature review for agile methodologies that discussed mobile applications development and what the researchers have concluded; the gap will be founded.

3) (The gap: there is no standardized model for developing mobile applications)

4) According to research's gap, research questions will be developed as well as proposing research framework.
5) Capability Maturity Model Integration (CMMI) will be used to provide guidance for developing research framework.

6) After preparing a proposed research framework, we need to test it based on real case studies.

7) In this step will get the results that will compare with other previous research studies.

8) Feedback: if the results don't meet the research goals, we need to improve the research framework (go back through step 3 to 6) to get final proposed framework.

\section{v. Research questions}

1) Are agile methodologies effects positively on mobile application development?

2) Are agile methodologies with CMMI effects positively on mobile application development?

3) Does proposed model affect positively on mobile application development?

4) Does the expected outcome have a significant effect on mobile application development?

\section{Conclusion}

According to literature review, there is no research study that provides detail reasons to use Agile as essential model for mobile application development for that reason, this research aims to encourage and contribute to prove that as well as helping developers in using a standardized model for developing mobile applications based on agile methodologies. 


\section{References}

1- [1] S. T. Acuña and X. Ferré, "Software Process Modelling," in ISAS-SCI (1), 2001, pp. 237-242.

2- [2] Y. B. Leau, et al., "Software Development Life Cycle AGILE vs Traditional Approaches," in International Conference on Information and Network Technology, 2012, pp. 162-167.

3- [3] O. Nikiforova, et al., "Integration of MDA Framework into the Model of Traditional Software Development," in DB\&IS, 2008, pp. 229-239.

4- [4] G. Milanov and A. NJEGUŠ, "Analysis of Return on Investment in Different Types of Agile Software Development Project Teams," Informatica Economica, vol. 16, pp. 7-18, 2012.

5- [5] G. Kumar and P. K. Bhatia, "Comparative Analysis of Software Engineering Models from Traditional to Modern Methodologies," in Advanced Computing \& Communication Technologies (ACCT), 2014 Fourth International Conference on, 2014, pp. 189-196.

6- [6] S. Balaji and M. Murugaiyan, "Waterfall vs. V-Model vs. Agile: A comparative study on SDLC," International Journal of Information Technology and Business Management, vol. 2, pp. 26-30, 2012.

7- [7] S. a. J. Khalaf and M. N. Al-Jedaiah, "Software quality and assurance in waterfall model and XP: a comparative study," WSEAS Transactions on Computers, vol. 7, pp. 1968-1976, 2008.

8- [8] H. K. Flora, et al., "Adopting an Agile Approach for the Development of Mobile Applications," International Journal of Computer Applications, vol. 94, pp. 43-50, 2014.

9- [9] A. Hammershoj, et al., "Challenges for mobile application development," in Intelligence in Next Generation Networks (ICIN), 2010 14th International Conference on, 2010, pp. 1-8.

10- [10] R. Holler, "Mobile application development: a natural fit with agile methodologies," VerisonOne LLC, Alpharetta, 2006.

11- [11] P. Abrahamsson, "Keynote: Mobile software development-the business opportunity of today," in Proceedings of the International Conference on Software Development, 2005, pp. 20-23.

12- [12] P. Abrahamsson, et al., "Mobile-D: an agile approach for mobile application development," in Companion to the 19th annual ACM SIGPLAN conference on Object-oriented programming systems, languages, and applications, 2004, pp. 174-175.

13- [13] Y.-J. Jeong, et al., "Development process of mobile application SW based on Agile methodology," in Advanced Communication Technology, 2008. ICACT 2008. 10th International Conference on, 2008, pp. 362-366. 